J. Gynäkol. Endokrinol. CH 2021 · 24:120-127 https://doi.org/10.1007/s41975-021-00193-0 Angenommen: 19. April 2021

Online publiziert: 4. Juni 2021

(c) Der/die Autor(en) 2021

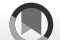

Jennifer-Christin Kuchernig

Schaper \& Brümmer GmbH \& Co. KG, Salzgitter, Deutschland

\title{
Sexuelle Unlust - Wirkmechanismen des natürlichen Aphrodisiakums Damiana (Turnera diffusa)
}

wird nicht durch eine organische Störung oder Krankheit verursacht, so liegt eine "hypoactive sexual desire disorder" (HSDD) vor. Die sexuelle Appetenzstörung HSDD ist die häufigste Form der FSD und wird definiert als anhaltend oder wiederholt auftretende/s mangelnde/s (oder fehlende/s) sexuelle/s Fantasien/Verlangen nach sexueller Aktivität, was deutliches Leiden oder zwischenmenschliche Schwierigkeiten verursacht $[5,6]$.

Sexuelle Unlust ist also nicht immer auch klinisch bedeutungsvoll, denn sie wird sehr unterschiedlich erlebt und bewertet. Wichtig ist der entstehende individuelle Leidensdruck, also jene Störungen der Libido, die zu einem persönlichen Leidenszustand und/oder einer partnerschaftlichen Belastung werden [7]. Besteht dieser jedoch, so zeigt sich, dass sexuelle Funktionsstörungen mit einer schlechten Lebensqualität verbunden sind, unter welcher Frauen umfassender und möglicherweise schwerwiegender leiden als Männer, dass sie sich aber nur zu 20-34\% aktiv Hilfe bei einem Arzt suchen [3, 8].

Dabei können stressige Lebensphasen, der demografische Hintergrund oder Probleme in der Partnerschaft genauso lustmindernde Effekte haben wie hormonelle Faktoren, z. B. ein durch die Einnahme der Pille ausgelöster Mangel an männlichen Hormonen, eine Entfernung der Eierstöcke, eine vorangegangene Schwangerschaft oder die Umstellung der Hormone in den Wechseljahren. Auch die Behandlung mit Blutdrucksenkern, Beruhigungsmitteln,
Antiöstrogenen, Neuroleptika oder Antidepressiva kann als Nebenwirkung $\mathrm{zu}$ einem verminderten Lustempfinden führen. Sexuelle Probleme können eine Folge, aber auch ein Warnsignal für eine schwerwiegende Grunderkrankung wie Diabetes, eine Infektion, Erkrankungen des Urogenitaltrakts oder Krebs sein. So können neben Erkrankungen des Unterleibes auch Nierenerkrankungen, Herzkrankheiten, Depressionen oder neurologische Erkrankungen auslösend sein.

Wird die Symptomatik thematisiert, so kann eine genauere Diagnose auch ohne tieferes Fachwissen mit den kurzen Verfahren „Decreased Sexual Desire Screener“ (DSDS) oder „Process Of Care“ (POC), die dem Erkennen von generalisiert erworbener HSDD bei Frauen dienen, erreicht werden $[4,9]$.

Optionen für einen Einstieg in die Behandlung gibt es viele. So können neben einer gezielten Anamnese und Untersuchung die Aufklärung über sexuelle $\mathrm{Ne}$ benwirkungen eines Medikaments und die Veränderungen der sexuellen Funktion im Zusammenhang mit den Wechseljahren sowie der Rat zu einer besseren Kommunikation mit Partnern hilfreich sein. Auch die Umstellung auf ein anderes Verhütungsmittel, die Dosisreduktion, das Absetzen oder Umstellen eines selektiven Serotonin- oder Serotonin- und Noradrenalin-Wiederaufnahmehemmers oder anderer Medikamente kann Abhilfe schaffen. Viele sexuelle Gesundheitsprobleme haben jedoch eine biopsychosoziale Ätiologie und erfordern möglicherweise eine multidiszipli- 


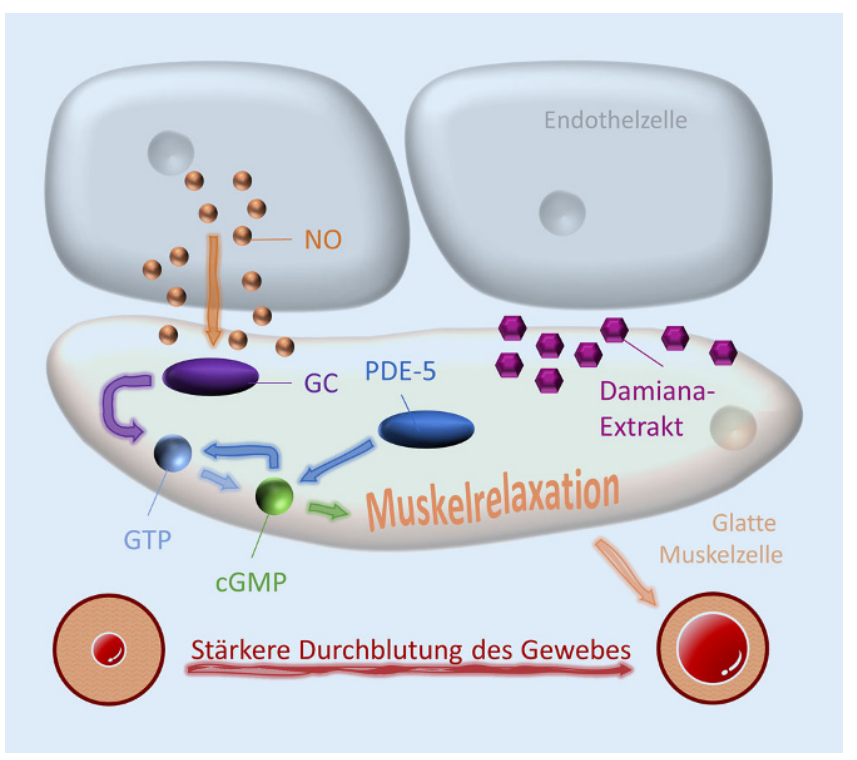

Abb. 1 ॥ „NO-cGMP pathway". Bei sexueller Stimulation kommt es zu einer Freisetzung von Stickstoffmonoxid (NO), aus den Endothelzellen und Diffusion in die glatten Muskelzellen. Das bioaktive Molekül NO stimuliert dann seinerseits das Enzym Guanylatzyklase (GC). Die GC wiederum bildet aus Guanosintriphosphat (GTP) zyklisches Guanosinmonophosphat (cGMP). Eine der wichtigsten Funktionen von cGMP ist die Relaxation glatter Muskelzellen, die zu einer stärkeren Durchblutung des Gewebes führt. cGMP wird im weiteren Verlauf der Reaktion durch das Enzym Phosphodiesterase Typ 5 (PDE-5) wieder in unwirksame Metaboliten abgebaut

näre Behandlung. Dann kann die Überweisung an andere Fachgebiete, wie z.B. Gynäkologie oder Urogynäkologie, Sexualmedizin, Sexualtherapie oder Psychotherapie angezeigt sein [4].

Pharmakotherapeutisch stehen nur relativ wenige Optionen zur Verfügung. Hierzu zählt einerseits die Off-LabelAnwendung von transdermalem Testosteron oder Dehydroepiandrosteron (DHEA). Andererseits kann ein möglicher Baustein der Behandlung in der Verwendung geeigneter Phytopharmaka liegen.

So ist die Pflanze Damiana (Turnera diffusa), besonders ihre Blätter, für ihre aphrodisierenden Eigenschaften seit sehr langer Zeit bekannt. Die erste Erwähnung ihrer Verwendung als Aphrodisiakum stammt bereits aus dem Jahr 1699, als der spanische Missionar Pater Juan Maria de Salvatierra über mexikanische Indianer berichtete, die ein Getränk aus Damiana-Blättern zubereiteten, das als Aphrodisiakum geschätzt wurde, indem sie sie in Wasser einweichten und Zucker hinzufügten [10]. Und auch Tierversuche zeigten eine Verbesserung des Sexualverhaltens sexuell erschöpfter Rat- ten sowie eine aphrodisierende Wirkung [11-15].

Aus diesen Gründen waren und sind Extrakte aus Damiana-Blättern auch immer wieder Gegenstand wissenschaftlicher Forschungen. So wurden in In-vitroStudien eine relaxierende Wirkung auf das Corpus cavernosum, eine Hemmwirkung auf das Enzym Phosphodiesterase Typ 5 (PDE-5), eine Hemmwirkung auf die Aromatase und eine östrogene Wirkung nachgewiesen. Außerdem wurden eine Angstminderung und Schmerzlinderung erzielt.

Der vollständige Wirkmechanismus von Damiana ist bis heute nicht bekannt, es wird jedoch angenommen, dass eine für pflanzliche Arzneimittel typische Gesamtwirkung aus den beobachteten Einzelwirkungen hervorgeht.

\section{Damiana (Turnera diffusa): aktueller Stand wissenschaft- licher Forschung}

Bei sexueller Stimulation kommt es zu einer Freisetzung von Stickstoffmonoxid (NO) aus den Endothelzellen und Diffusion in die glatten Muskelzellen. Das bioaktive Molekül NO stimuliert dann seinerseits das Enzym Guanylatzyklase (GC), wodurch wiederum aus Guanosintriphosphat (GTP) zyklisches Guanosinmonophosphat (cGMP) gebildet wird [16]. Eine der wichtigsten Funktionen von cGMP ist die Relaxation glatter Muskelzellen, die somit zu einer stärkeren Durchblutung des Gewebes führt (•Abb. 1). Diese Entspannung führt bei Männern, $a b$ einer gewissen Schwellenwertkonzentration von cGMP im Schwellkörper, zum Zustandekommen einer Erektion. Jedoch sind die Neurotransmitter für das Anschwellen der klitoralen Schwellkörper denen beim Mann sehr ähnlich, und die Freisetzung von NO hat auch hier eine besondere Bedeutung. Denn ähnlich der männlichen Reaktion führt die gesteigerte NOAktivität auch bei Frauen zu einer kavernösen und arteriellen glattmuskulären Relaxation und dadurch zu einer Anschwellung der Klitoris und der genitalen Region [16].

Eine solche entspannende Wirkung von Damiana aufdas Corpus cavernosum und somit eine Relaxation der glatten Muskulatur wurde für Dichlormethanund methanolische Extrakte unter Verwendung von L-Phenylephrin-vorkontrahierten Streifen des Corpus cavernosum von Meerschweinchen gezeigt. Bei Dosen von $10 \mathrm{mg} / \mathrm{ml}$ wurde eine Relaxation von $89 \%$ bzw. $86 \%$ beobachtet. Bei einer Dosis von $5 \mathrm{mg} / \mathrm{ml}$ betrugen die erhaltenen Werte $58 \%$ und $62 \% \mathrm{Re}$ laxation. Positivkontrollen mit Sildenafilcitrat in einem Dosisbereich von 0,35 bis $35 \mathrm{pg} / \mathrm{ml}$ ergaben mäßige Wirkungen bis zu $46 \%$ iger Relaxation bei $35 \mathrm{pg} / \mathrm{ml}$. Als Mechanismus hinter dieser Wirkung wurde von den Autoren eine Beeinflussung des „NO-cGMP pathway “ diskutiert [17].

Eine weitere Arbeitsgruppe analysierte, ob die durch $10 \mathrm{mg} / \mathrm{kgKG}$ Damiana entstehende und stärker als durch $10 \mathrm{mg} /$ kgKG Sildenafilcitrat ausgeprägte erhöhte sexuelle Motivation und die gesteigerte sexuelle Leistung sexuell träger Ratten über den NO-Weg ausgelöst werden. Außerdem untersuchte sie, ob solche Effekte auf der Ebene des Gehirns oder des Rückenmarks ausgeübt werden. Die Autoren kamen zu dem Schluss, dass die Ergebnisse die Annahme stützen, dass die 
Wirkung eines wässrigen Extrakts von Damiana die Beteiligung des NO-Weges auf zentraler und peripherer Ebene beinhaltet [13].

cGMP wird im weiteren Verlauf der Reaktion durch PDE-5 in unwirksame Metaboliten abgebaut (• Abb. 1). Die vermehrte Durchblutung kann somit durch Hemmung des cGMP-Abbaus mit PDE5-Inhibitoren verbessert werden. Auch in einem weiblichen Tiermodel führte Sildenafil, ein potenter Hemmstoff der cGMP-spezifischen PDE-5, zu einer erleichterten Genitalerregung [18]. Daher steht die durch Feistel et al. gezeigte PDE5-Hemmung durch Damiana-Extrakte gut im Einklang mit der durchblutungsfördernden Wirkung [19, 20].

So zeigten Feistel et al. für einen alkoholischen, gereinigten Trockenextrakt (basierend auf einem Rohextrakt mit $60 \%$ igem Ethanol) in vitro eine inhibitorische Aktivität auf PDE-5 mit einer mittleren inhibitorischen Konzentration $\left(\mathrm{IC}_{50}\right)$ von $5 \mu \mathrm{g} / \mathrm{ml}$ sowie für einen $90 \%$ igen ethanolischen Extrakt von Damiana einen $\mathrm{IC}_{50}$-Wert von $\sim 30 \mu \mathrm{g} / \mathrm{ml}$ $[19,20]$.

Auch Testosteron hat einen wesentlichen Einfluss auf die Durchblutung des Gewebes, denn das Androgen wirkt auf das Nervensystem, um die Erektion zu vermitteln. Das zur Gruppe der Stickoxide gehörende NO wird von der NOSynthase (NOS) gebildet, und es konnte nachgewiesen werden, dass die Bildung der NOS in nitrergen Nervenendigungen deutlich von der Höhe der Testosteronkonzentration abhängt [21]. Ist kein Testosteron vorhanden, kann es zu einer Herunterregulierung sowohl der Produktion als auch der Aktivität von NO kommen, wodurch die Reaktion auf periphere Stimulation über den NO-Weg verringert wird [22]. Ebenso ist die Expression von PDE-5 von Testosteron abhängig [21]. Aber nicht nur auf die Erektion, auch auf die Libido hat Testosteron einen großen Einfluss und wirkt auf diese fördernd. Die Wiederherstellung des normalen Testosteronspiegels bei Männern mit nachgewiesenen subnormalen Testosteronspiegeln verbesserte bei den meisten Probanden die Libido und bei mehr als $50 \%$ dieser Männer die erektile Funktion [23]. Und auch für die Libido

J. Gynäkol. Endokrinol. CH $2021 \cdot 24: 120-127$ https://doi.org/10.1007/s41975-021-00193-0 (c) Der/die Autor(en) 2021

\section{J.-C. Kuchernig}

Sexuelle Unlust - Wirkmechanismen des natürlichen Aphrodisiakums Damiana (Turnera diffusa)

\section{Zusammenfassung}

Sexuelle Unlust bei Frauen ist eine häufig auftretende und meist multifaktorielle Symptomatik in jedem Alter. Entsteht daraus ein individueller Leidensdruck, einhergehend mit einer schlechten Lebensqualität, dann leiden Frauen daran umfassender und möglicherweise schwerwiegender als Männer. Ihre sexuellen Probleme werden jedoch im klinischen Umfeld oft nicht erkannt und bleiben unbehandelt, denn nur 20-34\% der Betroffenen suchen aktiv Hilfe bei einem Arzt. Die Auslöser mangelnder Libido können vielfältig sein und neben stressigen Lebensphasen auch an bestehende Medikationen, den demografischen Hintergrund, Probleme in der Partnerschaft oder Umstellungen der Hormone z. B. in den Wechseljahren gekoppelt sein. Pharmakotherapeutisch stehen jedoch nur relativ wenige Optionen für die Behandlung zur Verfügung. Neben einer nur in der Off-Label-Anwendung mög- lichen Verabreichung von transdermalem Testosteron oder Dehydroepiandrosteron, der Überweisung an andere Fachgebiete oder einer interdisziplinären Zusammenarbeit kann ein möglicher Baustein der Behandlung in der Verwendung geeigneter Phytopharmaka liegen. Durch eine Gesamtwirkung der beobachteten Einzelwirkungen von Inhaltsstoffen der Pflanze Damiana (Turnera diffusa) aus der Familie der Safranmalven (Turneraceae) können daraus hergestellte Arzneimittel eine gute Behandlungsoption mit einem positiven Einfluss auf eine verminderte Libido darstellen.

\section{Schlüsselwörter}

Steigerung von sexuellem Verlangen. Mangelnde Libido - Weibliche sexuelle Funktionsstörungen · Vermindertes Lustempfinden . Pflanzliches Aphrodisiakum

\section{L'aversion sexuelle - mécanisme d'action de l'aphrodisiaque naturel Damiana (Turnera diffusa)}

Résumé

L'aversion sexuelle est une symptomatique fréquente et le plus souvent multifactorielle dans les femmes à tout âge. Si cela résulte dans un niveau de souffrance individuel accompagné d'une mauvaise qualité de vie, les femmes souffrent plus globalement et peut-être plus sévèrement que les hommes. Pourtant, leurs problèmes sexuels souvent ne sont pas reconnus dans le contexte médical et ne reçoivent pas de traitement parce que seulement $20 \%$ à $34 \%$ des personnes concernées cherchent l'assistance médical. Les déclencheurs d'une libido diminuée peuvent être divers et, outre des phases de la vie stressantes, ils peuvent aussi être couplés avec la médication existante, le contexte démographique, des problèmes dans le partenariat ou des changements hormonales, par exemple pendant la ménopause. Cependant, il y a relativement peu d'options pharmacothérapeutiques disponibles pour le traitement. Auprès d'une possible administration de testostérone ou de déhydroépiandrostérone transdermique, qui n'est possible que dans l'application hors AMM, le transfert vers d'autres domaines spécialisés ou une coopération interdisciplinaire, un autre élément possible du traitement peut être l'utilisation des produits phytopharmaceutiques appropriés. Vers un effet global des effets individuels observés de la plante Damiana (Turnera diffusa) de la famille des mauves safranées (Turnéracées), les médicaments dérivés de cette plante peuvent constituer une bonne option de traitement avec une influence positive sur une libido réduite.

Mots-clés

Augmentation du désir sexuel - Libido diminuée · Dysfonction sexuelle féminine . Plaisir diminué · Aphrodisiaque végétal 
Hier steht eine Anzeige.

\section{曾 Springer}




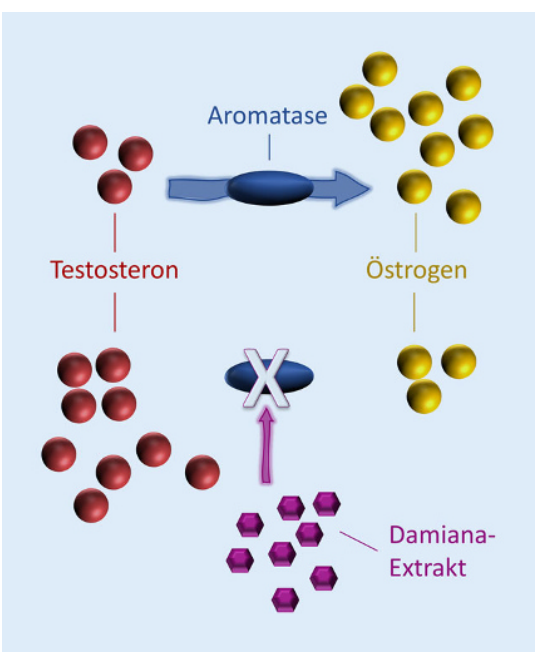

Abb. $2 \Delta$ Aromatasehemmung. Das Enzym Aromatase setzt Testosteron in Östrogen um. Eine Hemmung der Aromatase kann so zu einer Erhöhung des Testosteronspiegels im Blut führen

bei Frauen spielt Testosteron eine entscheidende Rolle. Androgene sind auch bei Frauen insbesondere bei der Stimulierung des sexuellen Motivationsverhaltens, der Aufrechterhaltung eines optimalen sexuellen Verlangens und möglicherweise bei der sexuellen Befriedigung wichtig. Zudem korreliert ein niedriger Testosteronspiegel mit einer verringerten Koitalfrequenz und einem Verlust des sexuellen Verlangens [24]. Normalerweise wird Testosteron durch sexualhormonbindendes Globulin (SHBG) oder Albumin gebunden, und nur $1-2 \%$ des gesamten Testosterons zirkulieren ungebunden. Im Allgemeinen wird angenommen, dass die nicht-SHBG-gebundene Fraktion biologisch aktiv ist [24].

Durch das Enzym Aromatase wird Testosteron in Östrogen umgesetzt (- Abb. 2). In diesem Kontext bringen die Ergebnisse von Zhao et al. [25] 2008 einen weiteren spannenden Ansatz für die Wirkmechanismen von Damiana mit sich.

Für einen methanolischen Extrakt zeigte sich eine dosisabhängig hemmende Wirkung gegenüber Aromatase (gewonnen aus Hep-2-Zellen). In einer Konzentration von $93,0 \mu \mathrm{g} / \mathrm{ml}$ war die Wirkung ähnlich stark wie bei $10 \mu \mathrm{M}$ der Positivkontrolle Aminoglutethimid, und der IC $_{50}$-Wert wurde auf $63,1 \mu \mathrm{g} / \mathrm{ml}$ bestimmt. Die Auswirkungen von 24 aus den Damiana-Blättern isolierten Einzelverbindungen auf die Aromataseaktivität wurden ebenfalls untersucht. Unter den 24 getesteten Verbindungen zeigten Pinocembrin und Acacetin mit IC $_{50}$-Wert von 10,8 bzw. $18,7 \mu \mathrm{M}$ die stärkste Hemmung. $10 \mu \mathrm{M}$ dieser aus dem Extrakt isolierten Komponenten unterdrückten die Aromataseaktivität zu 50,5\% bzw. 42,6\%. Da die sexuelle Funktion mit dem Testosteronspiegel in Verbindung steht und der Extrakt von Damiana die Aromataseaktivität signifikant unterdrücken kann, halten es die Autoren für möglich, dass die Einnahme von Extrakten aus dieser zu einer Erhöhung des Testosteronspiegels im Blut führen könnte [25].

In der gleichen Veröffentlichung konnte in einem Hefeöstrogenscreening (YES)-Test eine östrogene Wirkung für Damiana gezeigt werden. Es trat für den methanolischen Gesamtextrakt bei $250 \mu \mathrm{g} / \mathrm{ml}$ eine schwache Östrogenaktivität von $9 \%$ auf. Die isolierten Bestandteile Apigenin-7-Glucosid, Z-Echinacin und Pinocembrin zeigten eine östrogene Aktivität von $85 \%, 15 \%$ und $15,8 \%$ mit einer höchsten effektiven Dosis von $31 \mu \mathrm{M}, 125 \mu \mathrm{M}$ und $250 \mu \mathrm{M}$ und mit einer mittleren effektive (Wirk-)Konzentration $\left(\mathrm{EC}_{50}\right)$ von $10 \mu \mathrm{M}, 20 \mu \mathrm{M}$ und $67 \mu \mathrm{M}$, während die Positivkontrolle $17 \beta$-Estradiol einen $\mathrm{EC}_{50}$-Wert von 4,5 nM zeigte. Der $\mathrm{EC}_{50}$-Wert von $17 \beta$-Estradiol liegt somit mehr als den Faktor 2000 höher im Vergleich zu Apigenin-7-Glucosid [25].

Eine direkte hormonelle Wirkung von Damiana ist jedoch nicht zu erwarten. Denn ein 50 \%iger ethanolischer Extrakt $(\mathrm{g} / 10 \mathrm{ml})$ zeigte in intakten menschlichen Brustkrebszellen zwar eine Bindung von $6 \mu \mathrm{g}$ Progesteronäquivalenten $/ 2 \mathrm{~g}$ trockenes Kraut an intrazelluläre Rezeptoren für Progesteron (PR), jedoch keinen funktionellen Effekt: Er wurde daher als neutral eingestuft. Zudem konnte keine relevante Bindung an Estrogenrezeptoren (ER) nachgewiesen werden. Auch eine wachstumshemmende Wirkung, die nicht mit dem Vorhandensein von ER zusammenhängt, wurde nicht nachgewiesen [26].

Ist die sexuelle Unlust eher auf eine stressige Lebensphase oder innere
Anspannung zurückzuführen, so kann die anxiolytische Wirkung von Damiana hilfreich sein.

Diese angstlösende Wirkung, welche gleich in mehreren Veröffentlichungen gezeigt werden konnte, wird auf das enthaltene Apigenin (4,5,7-Trihydroxyflavon) zurückgeführt (• Tab. 1).

So zeigten Kumar et al., dass ein methanolischer Extrakt aus den oberirdischen Teilen von Damiana, unter Verwendung eines erhöhten Plus-LabyrinthTests und einer oralen Dosis von $25 \mathrm{mg} /$ $\mathrm{kgKG}$ eine mit $2 \mathrm{mg} / \mathrm{kgKG}$ Diazepam vergleichbare signifikant anxiolytische Wirkung aufwies. Die Aktivität nahm jedoch bei höheren Dosen ab, was die Autoren auf eine mögliche leichte Sedierung zurückführten. Der bioaktive Methanolextrakt wurde dann mit Petrolether, Chloroform und $n$-Butylalkohol ausgeschüttelt, und alle erhaltenen Fraktionen sowie der verbleibende Restmethanolextrakt wurden einzeln auf anxiolytische Aktivität untersucht. Dabei stellte sich heraus, dass die $n$-Butylalkohol-Fraktion und der verbleibende Restmethanolextrakt bei einer Dosis von $10 \mathrm{mg} / \mathrm{kgKG}$ bzw. $75 \mathrm{mg} / \mathrm{kgKG}$ signifikant anxiolytische Aktivität zeigten, für welche die Flavonoide/Alkaloide, die in der $n$-Butylalkohol-Fraktion enthalten sind, verantwortlich sein könnten. Der Gehalt an anxiolytischen Komponenten im Methanolextrakt war etwa 3-mal so hoch wie in der verbleibenden methanolischen Fraktion [27].

Die gleiche Arbeitsgruppe untersuchte zudem 3 ethanolische Urtinkturen (85\%ig) auf ihre anxiolytische Aktivität. Eine Urtinktur ist im Wesentlichen ein alkoholischer Extrakt, der gemäß den in der homöopathischen Pharmakopöe beschriebenen Verfahren hergestellt wird. Ebenfalls unter Verwendung eines erhöhten Plus-Labyrinth-Tests zeigten sich mit $2 \mathrm{mg} / \mathrm{kgKG}$ Diazepam vergleichbare, signifikante Wirkungen der Urtinkturen bei unterschiedlichen oralen Dosierungen (50 mg/kgKG, 75 mg/kgKG bzw. $125 \mathrm{mg} /$ $\mathrm{kgKG}$, je nach Hersteller). Solche Dosisunterschiede können in der Beimengung weiterer angstlösender Substanzen oder qualitativen und/oder quantitativen Verschiedenheit der Ausgangsmaterialien begründet sein. Auch in diesem Versuch 


\begin{tabular}{|c|c|c|c|}
\hline Referenz & Testsystem & Substanz/Ergebnis & $\begin{array}{l}\text { Vergleichssubstanz/ } \\
\text { Ergebnis }\end{array}$ \\
\hline Kumar et al. 2005 [27] & LACA-Mäuse & $\begin{array}{l}\text { Signifikant angstlösende Wirkung eines methanolischen Extraktes } \\
(25 \mathrm{mg} / \mathrm{kgKG})\end{array}$ & $\begin{array}{l}\text { Vergleichbar mit Diaze- } \\
\text { pam } 2,0 \mathrm{mg} / \mathrm{kgKG}\end{array}$ \\
\hline Kumar et al. 2005 [28] & LACA-Mäuse & $\begin{array}{l}\text { Signifikant angstlösende Wirkung } 3 \text { ethanolischer Urtinkturen ( } 85 \% \text { iger } \\
\text { Ethanol) }\end{array}$ & $\begin{array}{l}\text { Vergleichbar mit Diaze- } \\
\text { pam 2,0 mg/kgKG }\end{array}$ \\
\hline Kumar et al. 2006 [29] & LACA-Mäuse & $\begin{array}{l}\text { In einem methanolischen Extrakt }(250 \mathrm{~g}) \text { enthaltenes Apigenin }(2 \mathrm{mg} / \\
\mathrm{kgKG}) \text { ist verantwortlich für die angstlösende Wirkung }\end{array}$ & $\begin{array}{l}\text { Vergleichbar mit Diaze- } \\
\text { pam 2,0 mg/kgKG }\end{array}$ \\
\hline \multirow[t]{2}{*}{ Kumar et al. 2008 [30] } & \multirow[t]{2}{*}{ LACA-Mäuse } & Apigenin ( $2 \mathrm{mg} / \mathrm{kgKG}$ ) hat eine angstlösende Wirkung & $\begin{array}{l}\text { Vergleichbar mit Diaze- } \\
\text { pam } 2,0 \mathrm{mg} / \mathrm{kgKG}\end{array}$ \\
\hline & & Apigenin zeigt dosisabhängig exzellent schmerzlindernde Wirkung & $\begin{array}{l}\text { Vergleichbar mit Morphi- } \\
\text { um } 5 \mathrm{mg} / \mathrm{kgKG}\end{array}$ \\
\hline $\begin{array}{l}\text { Estrada-Reyes et al. } \\
2013 \text { [13] }\end{array}$ & Wistar-Ratten & $\begin{array}{l}\text { Ein wässriger Extrakt ( } 10 \mathrm{mg} / \mathrm{kgKG}) \text { erzeugte bei männlichen Ratten } \\
\text { eine anxiolytische Wirkung }\end{array}$ & - \\
\hline
\end{tabular}

nahm die Aktivität bei höheren Dosen wahrscheinlich aufgrund einer dann einsetzenden sedativen Wirkung ab. Alle 3 Urtinkturen wiesen in einem phytochemischen Screening ähnliche Klassen an pflanzlichen Inhaltsstoffen auf. Die Autoren schlossen aus diesem Screening, dass Flavonoide, Alkaloide oder Steroide für die anxiolytische Aktivität verantwortlich sein könnten [28].

Aber auch ein wässriger Extrakt von Damiana (10 mg/kgKG) konnte eine anxiolytische Wirkung erzeugen [13].

Weitere Forschung zeigte, dass für die anxiolytische Wirkung (vergleichbar mit 2,0 mg/kgKG Diazepam) eines methanolischen Extraktes aus Damiana (250 g) das enthaltene Apigenin $(2 \mathrm{mg} / \mathrm{kgKG})$ verantwortlich ist. Ziel der Untersuchung war es, den/die bioaktiven Bestandteil(e) aus Damiana unter Verwendung einer bioaktivitätsgesteuerten Fraktionierung $\mathrm{zu}$ isolieren. Hierfür wurde der methanolische Extrakt mit einem Lösungsmittelgradienten (Chloroform, Chloroform/ Methanol, Methanol/Acetonitril) über Kieselgel fraktioniert, und die 130 erhaltenen Fraktionen wurden entsprechend ihren Dünnschichtchromatographieprofilen zu 7 Fraktionen (F1-F7) gepoolt. Von diesen zeigte nur eine Fraktion (F5) signifikant anxiolytische Wirkung $(10 \mathrm{mg} / \mathrm{kgKG})$. In einem phytochemischen Screening konnten in dieser Fraktion Flavonoide und Alkaloide nachgewiesen werden. Diese Fraktion wurde erneut in 7 Unterfraktionen aufgetrennt, von welchen nur Fraktion 5.3 die signifikant anxiolytische Wirkung (5 mg/kgKG) zeigte und ausschließlich Flavonoide enthielt. Eine Dünnschichtchromatographie (Toluen:Chloroform:Aceton; 8:5:7) von Fraktion 5.3 ergab im Weiteren 2 isolierbare Reinsubstanzen (K1 und K2). Nur die Substanz K1 (2 mg/kgKG) zeigte eine signifikant anxiolytische und mit 2,0 mg/kgKG Diazepam vergleichbare Wirkung. Sie wurde als Apigenin (Schmelzpunkt, UV und NMR-Spektrum, Referenzvergleich) identifiziert und dieses somit von den Autoren als die Substanz mit angstlösender Aktivität in Damiana festgestellt [29].

In einer nachfolgenden Untersuchung wurde Apigenin als bioaktives Prinzip von Damiana von der gleichen Arbeitsgruppe einer genaueren pharmakologischen Bewertung unterzogen. Es zeigte sich, dass Apigenin ( $2 \mathrm{mg} / \mathrm{kgKG})$ signifikant die Anzahl der "head-dippings“ im Lochbrett-Test erhöhte sowie die Zeit bis zum Verlassen des hellen Areals und die Aufenthaltszeit im hellen Areal im Licht-Dunkel-Test verlängerte. Die Zeit bis zum Betreten eines Spiegelzimmers konnte gesenkt werden, und die Aufenthaltszeit im bzw. die Anzahl der Eintritte in das Spiegelzimmer erhöhte sich (jeweils vergleichbar mit 2,0 mg/kgKG Diazepam). Mit all diesen Beobachtungen bestätigten die Autoren die anxiolytische Aktivität von Apigenin. Wiederholt präsentierte sich auch die Dosisabhängigkeit der Wirkung und zeigte, dass bei 12 -fach erhöhter Dosierung $(25 \mathrm{mg} /$ $\mathrm{kgKG}$ ) leicht sedierende Effekte auftraten, nicht jedoch bei geringerer Dosierung $(5,10 \mathrm{mg} / \mathrm{kgKG})$ und auch nicht vergleichbar mit Diazepam (10 mg/kgKG). Apigenin zeigte dosisabhängig in diesen
Versuchen zudem exzellent schmerzlindernde Wirkung vergleichbar zu Morphium $(5 \mathrm{mg} / \mathrm{kgKG})$ mit einem maximalen Effekt nach $30 \mathrm{~min}$ (10 mg/kgKG). Dieser bleibt für $4 \mathrm{~h}$ signifikant erhalten [30].

Bekannt ist, dass Flavonoide, wie z.B. auch Apigenin, durch eine Vielzahl von Wechselwirkungen mit verschiedenen Rezeptoren und Signalsystemen, einschließlich $\gamma$-Aminobuttersäure (GABA)-Rezeptoren (• Abb. 3), eine Wirkung auf das Zentralnervensystem (ZNS) haben, $u$. a. auch eine angstlösende Wirkung. Die natürlich vorkommende Aminosäure GABA ist der primäre inhibitorische Neurotransmitter im Gehirn von Säugetieren, der von bis zu $40 \%$ der Neuronen freigesetzt wird. Apigenin wird als negativer Modulator der GABAWirkung beschrieben und hat komplexe modulatorische Wirkungen auf $\mathrm{GABA}_{\mathrm{A}^{-}}$ Rezeptoren [31]. Eine weiterer Mechanismus für die Wirkung von Apigenin ist eine verringerte Aktivierung von N-Methyl-D-Aspartat(NMDA)-Rezeptoren durch L-Glutaminsäure [31]. Denn Apigenin hat nicht nur eine antagonistische Wirkung auf GABA-, sondern auch auf NMDA-Kanäle, und die hemmende Wirkung auf die glutamaterge Übertragung könnte die in vivo berichteten beruhigenden Wirkungen erklären [32]. Apigenin scheint somit die Funktion des inhibitorischen GABA-Systems zu verstärken und gleichzeitig die Wirkung des exzitatorischen Glutamatsystems zu reduzieren.

In Übereinstimmung mit den Erkenntnissen zu diesen Wirkmechanismen ließen sich bei einer 31-jährigen 


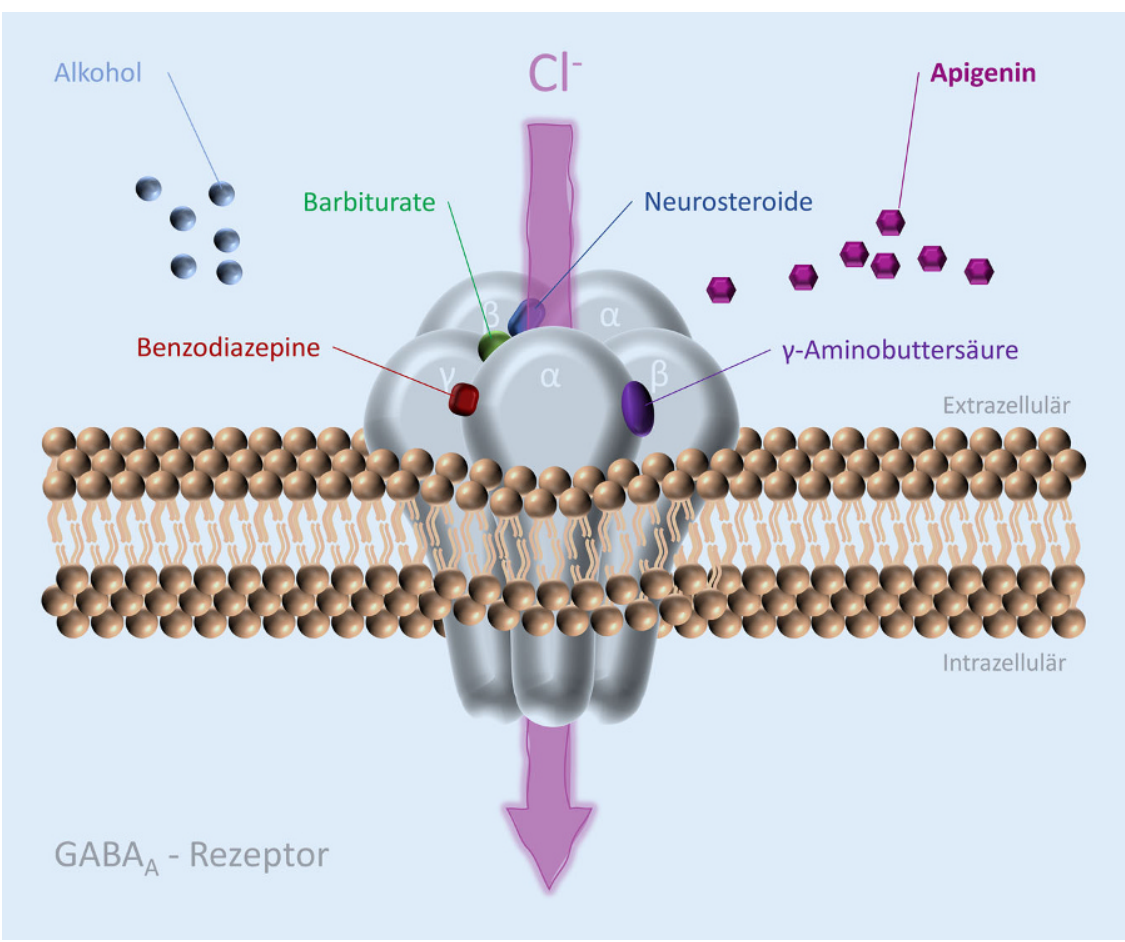

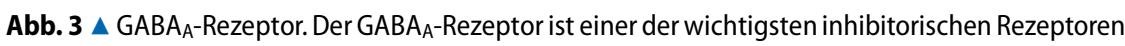
im Zentralnervensystem. Neben Bindungsstellen für -Aminobuttersäure (GABA) besitzt er zudem Bindungsstellen für Barbiturate, Neurosteroide und Benzodiazepine

Frau nach Einnahme eines aus Damiana hergestellten Arzneimittels (Remisens ${ }^{\circledR}$, Schaper \& Brümmer GmbH \& Co. KG, Salzgitter, Deutschland) entsprechende Veränderungen der elektrischen Aktivität des Gehirns nachweisen. Hierfür wurden vor und 90 min nach der Einnahme $(225 \mathrm{mg}$ Trockenextrakt, DEV $=5$ 7:1, Ethanol $90 \%$ [V/V]) die Hirnaktivitäten im entspannten Zustand und während verschiedener kognitiver und emotionaler Herausforderungen mittels EnkephaloVision untersucht (u.a. ein d2-Konzentrations- und Ausdauertest, ein Gedächtnistest, ein Bildvergleich, ein Stroop-Test und das Anschauen erotischer Bilder sowie eines erotischen „Chippendale“-Videos). Nach der Einnahme von Damiana und im entspannten Zustand zeigte sich ein Anstieg der spektralen $\alpha$-Leistung im Zentrum und in der parietalen Region des Kortex sowie im okzipitalen Kortex des Gehirns, welcher als Ausdruck eines höheren Entspannungszustands und weniger Angst interpretiert werden kann. Zudem wurden während der Durchführung des d2Konzentrations- und Ausdauertests eine Zunahme der spektralen $\delta$-Leistung im Frontalhirn und eine Abnahme der $\alpha$-Spektral-Leistung in den Zentral- und Parietallappen beobachtet. Während der Betrachtung erotischer Bilder stiegen die $\delta$ - und $\theta$-Spektral-Leistung in frontalen und temporalen Regionen. Ähnliche Ergebnisse wurden beim Betrachten des erotischen Videos erhalten. Eine Erhöhung der spektralen Leistung von $\delta$ und $\theta$ weist auf einen höheren Zustand der mentalen Aktivierung hin [33].

Zusammenfassend zeigen Extrakte aus Damiana (Turnera diffusa) verschiedene Wirkmechanismen, welche einen positiven Beitrag bei der Behandlung der unterschiedlichen Ursachen sexueller Unlust bei Frauen leisten können.

\section{Fazit für die Praxis}

Die vorgestellten Wirkmechanismen geben gute Hinweise darauf, dass Arzneimittel aus Damiana einen positiven Einfluss auf eine verminderte Libido nehmen können. Apigenin scheint hierbei ein wirksamkeitsbestimmender Bestandteil der anxiolytischen und schmerzlindernden Aktivität zu sein. Beide Aktivitäten können eine wichtige
Rolle spielen, und gerade die angstlösenden Effekte können helfen den Kopf zu befreien. Darüber hinaus führt der Gesamtextrakt zu einer Relaxation der glatten Muskulatur und somit, vermutlich über den NO-cGMP pathway, zu einer durchblutungsfördernden Wirkung, welche ihren Beitrag über die Intensivierung der Erregung leisten kann. Des Weiteren kann die aromatasehemmende Wirkung des Gesamtextraktes durch die Steigerung freien Testosterons zur Stärkung der Lust führen. Als phytotherapeutisch zugelassenes Arzneimittel in entsprechender Dosierung ( $z$. B. Remisens ${ }^{\circledR}$, eine Filmtablette dreimal täglich) kann ein Therapieversuch somit sinnvoll sein oder weitere Maßnahmen als Baustein der Therapie ergänzen.

\section{Korrespondenzadresse}

Dr. Jennifer-Christin Kuchernig

Schaper \& Brümmer GmbH \& Co. KG Bahnhofstraße 35, 38259 Salzgitter, Deutschland

Jennifer-C.Kuchernig@schaper-bruemmer.de

\section{Einhaltung ethischer Richtlinien}

Interessenkonflikt. J.-C. Kuchernig ist Mitarbeiterin der Schaper \& Brümmer GmbH \& Co. KG.

Für diesen Beitrag wurden von der Autorin keine Studien an Menschen oder Tieren durchgeführt. Für die aufgeführten Studien gelten die jeweils dort angegebenen ethischen Richtlinien.

Open Access. Dieser Artikel wird unter der Creative Commons Namensnennung 4.0 International Lizenz veröffentlicht, welche die Nutzung, Vervielfältigung, Bearbeitung, Verbreitung und Wiedergabe in jeglichem Medium und Format erlaubt, sofern Sie den/die ursprünglichen Autor(en) und die Quelle ordnungsgemäß nennen, einen Link zur Creative Commons Lizenz beifügen und angeben, ob Änderungen vorgenommen wurden.

Die in diesem Artikel enthaltenen Bilder und sonstiges Drittmaterial unterliegen ebenfalls der genannten Creative Commons Lizenz, sofern sich aus der Abbildungslegende nichts anderes ergibt. Sofern das betreffende Material nicht unter der genannten Creative Commons Lizenz steht und die betreffende Handlung nicht nach gesetzlichen Vorschriften erlaubt ist, ist für die oben aufgeführten Weiterverwendungen des Materials die Einwilligung des jeweiligen Rechteinhabers einzuholen.

Weitere Details zur Lizenz entnehmen Sie bitte der Lizenzinformation auf http://creativecommons.org/ licenses/by/4.0/deed.de. 


\section{Literatur}

1. World Health Organisation (WHO) (2011) WHO Europa - Definition Sexuelle und reproduktive Gesundheit. https://www.euro. who.int/de/health-topics/Life-stages/sexualand-reproductive-health/news/news/2011/06/ sexual-health-throughout-life/definition. Zugegriffen: 18.02.2021

2. Briken $P$ et al (2020) Estimating the prevalence of sexual dysfunction using the new ICD-11 guidelines. Dtsch Arztebl Int 117(39):653-658

3. Laumann EO, Paik A, Rosen RC (1999) Sexual dysfunction in the United States: prevalence and predictors. JAMA 281(6):537-544

4. Parish SJ et al (2019) the international society for the study of women's sexual health process of care for the identification of sexual concerns and problems in women. in mayo clinic proceedings. Elsevier,

5. Naumann G et al (2010) Was ist HSDD (Hypoactive Sexual Desire Disorder, Störung mit vermindertem sexuellen Verlangen)? Geburtshilfe Frauenheilkd 70(09):701-706

6. Brotto LA (2010) The DSM diagnostic criteria for hypoactive sexual desire disorder in women. Arch SexBehav 39(2):221-239

7. Bitzer J (2004) Libidostörungen bei der Frau. Teil 1: Definitionen und Abklärung. Forum Médical Suisse. EMH Media,

8. Ahrendt H-J, Adolf D, Friedrich C (2011) Inzidenz sexueller Probleme in der gynäkologischen Praxis. Sexuologie 18(1):25-29

9. Clayton AH et al (2009) Validation of the decreased sexual desire screener (DSDS): a brief diagnostic instrument for generalized acquired female hypoactive sexual desire disorder (HSDD). J Sex Med 6(3):730-738

10. Tyler VE (1983) Damiana - history of a herbal hoax. Pharm Hist 25(2):55-60

11. Estrada-Reyes R et al (2009) Turnera diffusa wild (Turneraceae) recovers sexual behavior in sexually exhausted males. J Ethnopharmacol 123(3):423-429

12. ArlettiRetal(1999)Stimulating propertyofTurnera diffusa and Pfaffia paniculata extracts on the sexual behavior of male rats. Psychopharmacology 143(1):15-19

13. Estrada-Reyes R, Carro-Juárez M, Martínez-Mota L (2013) Pro-sexual effects of Turnera diffusa wild (Turneraceae) in male rats involves the nitric oxide pathway. JEthnopharmacol 146(1):164-172

14. Kumar S, Madaan R, Sharma A (2009) Evaluation of aphrodisiac activity of Turnera aphrodisiaca. Int J Pharmacogn Phytochem Res 1:1-4

15. Estrada-Reyes R et al (2016) Prosexual effect of Chrysactinia mexicana A. Gray (Asteraceae), false Damiana, in a model of Male sexual behavior. Biomed Res Int 2016:1-9. https://doi.org/10.1155/ 2016/2987917

16. Suttorp NB, Möckel M, Siegmund B, Dietel M (2016) Harrisons Innere Medizin. ABW Wissenschaftsverlag

17. Hnatyszyn 0 etal (2003) Argentinian plant extracts with relaxant effect on the smooth muscle of the corpus cavernosum of Guinea pig. Phytomedicine 10(8):669-674

18. Min Ket al (2000) Sildenafil augments pelvic nervemediated female genital sexual arousal in the anesthetized rabbit. Int JImpot Res 12(3):S32-S39

19. Feistel $B$ (2010) Damiana (Turnera diffusa wild.) traditionally used aphrodisiac as modern PDE-5 inhibitor. Planta Med 76:P2
20. Feistel B, Appel K, Pischel I (2018) Traditional Turnera diffusa WILLD. - new pharmacological aspects about Damiana. in. Phytokongress, Wien, Österreich, 2018 Phytotherapie, Austria

21. Unbekannt (2007) Welche Rolle spielt Testosteron bei der erektilen Funktion? https://www. andrologen.info/andros/andrologie/ED/print/T_ ED.pdf.Zugegriffen: 19.05.2021

22. Baba K et al (2000) Delayed testosterone replacement restores nitric oxide synthase-containing nerve fibres and the erectile response in rat penis. BJUInt 85(7):953-958

23. Yassin AA, Saad F (2007) Endocrinology: improvement of sexual function in men with late-onset Hypogonadism treated with testosterone only. JSexMed 4(2):497-501

24. Davis SR, Tran J (2001) Testosterone influences libido and well being in women. Trends Endocrinol Metab 12(1):33-37

25. Zhao J et al (2008) Anti-aromatase activity of the constituents from damiana (Turnera diffusa). JEthnopharmacol 120(3):387-393

26. Zava DT, Dollbaum CM, Blen M (1998) Estrogen and Progestin Bioactivity of Foods, Herbs, and Spices. Proc Soc Exp Biol Med 217(3):369-378

27. Kumar S, Sharma A (2005) Anti-anxiety activity studies of various extracts of Turnera aphrodisiaca ward. WHER 5(4):13-21

28. Kumar S, Sharma A (2005) Anti-anxiety activity studies on Homoeopathic formulations of Turnera aphrodisiaca ward. Evidence-based complementary and alternative. Medicine 2(1):117-119

29. Kumar S, Sharma A (2006) Apigenin: The Anxiolytic Constituent of Turnera aphrodisiaca. Pharm Biol 44(2):84-90

30. Kumar S, Madaan R, Sharma A (2008) Pharmacological evaluation of Bioactive Principle of Turnera aphrodisiaca. Indian J Pharm Sci 70(6):740-744

31. Hinton T, Hanrahan JR, Johnston GA (2017) Flavonoid actions on receptors for the inhibitory neurotransmitter GABA. Flavonoids-from biosynthesis to human health. InTech,

32. Losi G et al (2004) Apigenin modulates GABAergic and glutamatergictransmission in cultured cortical neurons. Eur JPharmacol 502(1):41-46

33. Dimpfel W, Schombert L (2017) Effect of Damiana extract on the electric activity of a female brain. The 21th International Congress Phytopharm, Graz. Reviews of clinical pharmacology and drug therapy, $\mathrm{S} 18$

Hinweis des Verlags. Der Verlag bleibt in Hinblick auf geografische Zuordnungen und Gebietsbezeichnungen in veröffentlichten Karten und Institutsadressen neutral.

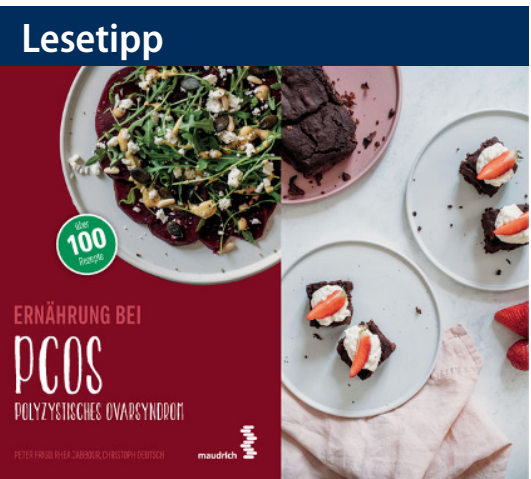

\section{Genussvoll essen bei PCOS}

Das Polyzystische Ovarsyndrom ist eine der häufigsten hormonellen Störungen bei Frauen. Mit der richtigen Ernährungsweise lässt es sich jedoch gut behandeln. Oft kann die Ernährungstherapie nicht nur die Fruchtbarkeit wiederherstellen, sondern auch Folgeerkrankungen verhindern.

Dieses Buch zeigt, wie Sie die nötigen Veränderungen unkompliziert in Ihren Alltag einbauen - mit vielen praktischen Tipps und zahlreichen Rezepten, vom Frühstück über Hauptspeisen mit und ohne Fleisch bis zum köstlichen Dessert.

- Über 100 abwechslungsreiche Rezepte

- Hilfreiche Infos zu Diagnose und Therapie

- Passende Ernährungsformen im Überblick

- Tipps zu Food Tracking und Ernährungshelfern

- Gratis Einkaufslisten-App

Peter Frigo, Rhea Jabbour, Christoph Deutsch. Ernährung bei PCOS. Polyzystisches Ovarsyndrom. Verlag Facultas / Maudrich, 2021

Erhältlich als Taschenbuch (ISBN 978-399002-124-8) und als E-Book (EPUB) (9783-99111-344-7) 